\title{
Wavelength-resolution SAR Incoherent Change Detection Based on Image Stack
}

\author{
Viet Thuy Vu, IEEE Member
}

\begin{abstract}
The paper presents a wavelength-resolution SAR incoherent change detection method based on image stack, i.e. there are more than one reference or/and surveillance image. Considering image stack in statistical hypothesis test for change detection is expected to result into a simple mathematical expression for implementation and provide better change detection results. As presented in this paper, a statistical hypothesis test is developed on bivariate Gaussian distribution for an image stack of two reference and one surveillance images. The requirement for the image stack is three images associated with three measurements with no change between two of them. A detection method with simple processing scheme is proposed. The method is experimented with 24 CARABAS data sets. The results indicate that high average detection probability, e.g. $96 \%$, with very low false alarm rate, e.g. only 0.19 per square kilometer, is obtained with the proposal.
\end{abstract}

Index Terms-SAR, change detection, statistics, bivariate Gaussian distribution

\section{INTRODUCTION}

Wavelength-resolution SAR change detection has been researched for more than a decade. One of the first measurement campaigns with CARABAS is reported in [1],[2]. The advantages of wavelength-resolution SAR change detection can be listed as follows. For the wavelength of several meters, the contribution of small scatterers to radar echoes is not significant. The main contribution comes from large objects with meter size or larger. Such large scatterers are usually stable in time and less sensitive to environmental effects such as wind, rain, and snow. The use of ultrawideband signal allows reducing statistical fluctuations in SAR images even with different incident geometries. Such characteristics help to simplify change detection algorithms or methods.

The idea of wavelength-resolution SAR incoherent change detection based on image stack is introduced in [3] for the first time as an approach for the problem of elongated structures that are sensitive to flight path. The elongated structures such as power lines and fences which stand out from the background usually cause false alarms in wavelength-resolution SAR change detection. With an image stack, the false alarms caused by the elongated structures are reduced significantly with the well-known adaptive processing mechanism, i.e. adaptive noise canceler. The requirement for the image stack is three images associated with three measurements with no change between two of them. It is worth to mention that SAR change detection based on image stack at regular intervals of

Viet T. Vu is with Blekinge Institute of Technology, Karlskrona, 37179 Sweden, Email: viet.thuy.vu@bth.se

Manuscript received January 23, revised March 23,2017. time has been experimented. The systems like MODIS can provide high temporal resolution data [4]. The applications can be found in e.g. detection of beetle infestation in pine forests [5].

The objective of the paper is to introduce a wavelengthresolution SAR incoherent change detection method based on image stack. The method is developed on statistical hypothesis test with consideration of all images in image stack. The requirement for the image stack is also three images associated with three measurements with no change between two of them. The experiments with 24 CARABAS data sets are also provided to verify and evaluate the proposal.

The rest of the paper is organized as follows. Section 2 presents the formulation of the statistical hypothesis tests with the consideration of image stack. The wavelength-resolution SAR incoherent change detection method is introduced in Section 3. Section 4 provides the experimental results for verification and evaluation of the proposal. Section 5 gives conclusions.

\section{Statistical Hypothesis Tests with CONSIDERATION OF IMAGE STACK}

Similar nomenclatures that have been used in [1] and [3] are adopted here to formulate the expressions of statistical hypothesis test for change detection.

\section{A. Scenario}

We assume here that a ground scene is illuminated by three passes of a SAR system. The flight paths of three illuminations are identical. The time separation between the illuminations 1 and 2 is small enough so that the ground scene is considered to be unchanged. This is especially true for wavelength-resolution SAR as mentioned in the introduction. The changes can occur between the illuminations 1 and 3 due to, e.g. deforestation, movements of ground objects like vehicles, illegal activities or other military and civilian activities which must be controlled. The collected data in the illuminations are then used to form three complex SAR images and they are co-registered. After removing the phase, the magnitude images are denoted by matrices $\mathbf{Z}_{1}, \mathbf{Z}_{\mathbf{2}}$ and $\mathbf{Z}_{\mathbf{3}}$. Under the assumption of no change, the subtraction of $\mathbf{Z}_{2}$ to $\mathbf{Z}_{1}$ results into the clutter or false alarms $\mathbf{C}_{\mathbf{r}}$ and noise $\mathbf{N}_{\mathbf{r}}$

$$
\mathbf{Z}_{2}-\mathbf{Z}_{1}=\mathbf{Z}_{\mathrm{r}}=\mathbf{C}_{\mathrm{r}}+\mathbf{N}_{\mathrm{r}}
$$

If there is no change occurred between the illuminations 1 and 3, the subtraction of $\mathbf{Z}_{\mathbf{3}}$ to $\mathbf{Z}_{\mathbf{1}}$ will also result into only clutter 


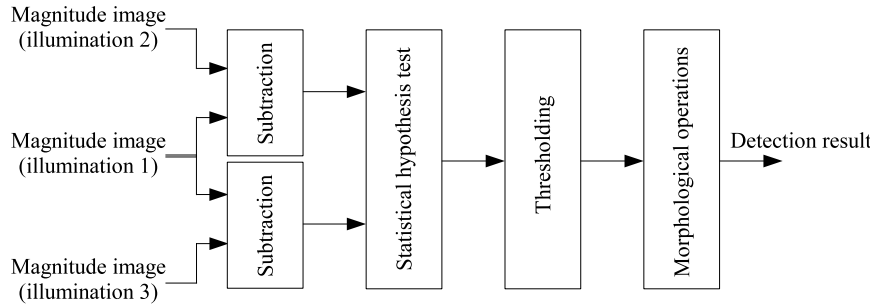

Fig. 1. Processing scheme for wavelength-resolution SAR incoherent change detection based on image stack.

$\mathbf{C}_{\mathbf{u}}$ and noise $\mathbf{N}_{\mathbf{u}}$

$$
\mathbf{Z}_{3}-\mathbf{Z}_{\mathbf{1}}=\mathbf{Z}_{\mathbf{u}}=\mathbf{C}_{\mathbf{u}}+\mathbf{N}_{\mathbf{u}}
$$

Conversely, if there are changes occurred between the illuminations 1 and 3, the subtraction of $\mathbf{Z}_{\mathbf{3}}$ to $\mathbf{Z}_{\mathbf{1}}$ will result into the changes $\mathbf{S}_{\mathbf{u}}$, clutter $\mathbf{C}_{\mathbf{u}}$ and noise $\mathbf{N}_{\mathbf{u}}$

$$
\mathbf{Z}_{\mathbf{u}}=\mathbf{S}_{\mathbf{u}}+\mathbf{C}_{\mathbf{u}}+\mathbf{N}_{\mathbf{u}}
$$

Equation (2) corresponds to a hypothesis $H_{0}$ which considers no change between the illuminations 1 and 3 . Equation (3) is associated with a hypothesis $H_{1}$ considering the changes.

\section{B. Statistical hypothesis test}

Let's consider a vector $\mathbf{z}=\left[z_{r}, z_{u}\right]^{T}$ where $z_{r}$ is a data sample of $\mathbf{Z}_{\mathbf{r}}$ and $z_{u}$ is the corresponding sample of $\mathbf{Z}_{\mathbf{u}}$ and let the vectors $\mathbf{s}, \mathbf{c}$ and $\mathbf{n}$ denote the change, clutter, and noise signals, respectively

$$
\mathbf{s}=\left[\begin{array}{c}
s_{r} \\
s_{u}
\end{array}\right] \quad \mathbf{c}=\left[\begin{array}{c}
c_{r} \\
c_{u}
\end{array}\right] \quad \mathbf{n}=\left[\begin{array}{l}
n_{r} \\
n_{u}
\end{array}\right]
$$

In the change signal vector, $s_{r}=0$ while $s_{u} \geq 0$. The hypotheses $H_{0}$ and $H_{1}$ are formulated by

$$
\begin{aligned}
& H_{0}: \mathbf{z}=\mathbf{c}+\mathbf{n}=\left[\begin{array}{c}
c_{r}+n_{r} \\
c_{u}+n_{u}
\end{array}\right] \quad \text { (no change) } \\
& H_{1}: \mathbf{z}=\mathbf{s}+\mathbf{c}+\mathbf{n}=\left[\begin{array}{c}
c_{r}+n_{r} \\
s_{u}+c_{u}+n_{u}
\end{array}\right] \text { (change) }
\end{aligned}
$$

The Neyman-Pearson lemma states that when performing a hypothesis test between two simple hypotheses $H_{0}$ and $H_{1}$, the likelihood-ratio test which rejects $H_{1}$ in favor of $H_{0}$ when

$$
\Lambda(\mathbf{z})=\frac{P\left(\mathbf{z} \mid H_{1}\right)}{P\left(\mathbf{z} \mid H_{0}\right)} \leq \lambda
$$

The statistical analysis about stability of wavelengthresolution SAR images in [6] based on bivariate distribution shows that the subtractions (1), (2) and (3) result into Gaussian distributed clutter. The analysis also shows that the noise mainly consists of the thermal noise and, therefore, it is not necessary to consider speckle noise in wavelength-resolution SAR change detection. In other words, the noise $n_{r}$ and $n_{u}$ are justified to be modeled by Gaussian processes. The clutter $c_{r}$ and $c_{u}$ are also modeled by different Gaussian processes. Since the sum of the two Gaussian processes is another Gaussian process, modeling clutter plus noise by a Gaussian process is reasonable. The conditional probability under the hypothesis $H_{0}$ will be a joint probability of two Gaussian processes, i.e. bivariate Gaussian process. The probability density function $P\left(\mathbf{z} \mid H_{0}\right)$ of a bivariate Gaussian process is defined by (7) where $\mu$ and $\sigma$ denote expected value and standard deviation of a variable $\mathbf{Z}_{(\cdot)}$, respectively, $\rho$ is the correlation coefficient between the variables $\mathbf{Z}_{\mathbf{r}}$ and $\mathbf{Z}_{\mathbf{u}}$. In the case changes occurred between the illuminations 1 and 3 , the probability density function of $P\left(\mathbf{z} \mid H_{1}\right)$ is given by (8). The statistical hypothesis test (6) is rewritten in following form

$$
\Lambda(\mathbf{z})=\exp \left(\frac{s_{u}\left(2 z_{u}-2 \mu_{u}-s_{u}\right)}{2 \sigma_{u}^{2}\left(1-\rho^{2}\right)}-\frac{\rho s_{u}\left(z_{r}-\mu_{r}\right)}{\sigma_{r} \sigma_{u}\left(1-\rho^{2}\right)}\right)
$$

or we can even rewrite as

$$
\ln (\Lambda(\mathbf{z}))=\mathbf{s}^{T} \boldsymbol{\Sigma}^{-1}(\mathbf{z}-\mu)-\frac{1}{2} \mathbf{s}^{T} \boldsymbol{\Sigma}^{-1} \mathbf{s}
$$

where the covariance matrix $\boldsymbol{\Sigma}$ is given by

$$
\boldsymbol{\Sigma}=\left[\begin{array}{cc}
\sigma_{r}^{2} & \rho \sigma_{r} \sigma_{u} \\
\rho \sigma_{r} \sigma_{u} & \sigma_{u}^{2}
\end{array}\right]
$$

The likelihood ratio test (9) or logarithmic one (10) shows the problem that we need to assume the magnitudes of the changes $s_{u}$ to compute the test statistic. If we set $s_{u}$ too high, there is a high possibility that several changes with low radar cross-section are either undetected or detected with very high false alarm rate as the probability of the magnitude $z_{u}-\mu_{u}$ (right tail) can be larger than the probability of the magnitude $z_{u}-\mu_{u}-s_{u}$ (left tail). Conversely, if $s_{u}$ is set too low, the values of the statistical hypothesis test change insignificantly with respect to magnitudes. This might result into high false alarm rate. One way to estimate $s_{u}$ is to base on the Gaussian cumulative distribution function as presented in [3].

$$
s_{u}=\mu_{u}+\sigma_{u} \sqrt{2} \operatorname{erfc}^{-1}\left[2 P\left(z_{u}>s_{u}\right)\right]
$$

where we can set the probability $P\left(z_{u}>s_{u}\right)$ as desired.

\section{WAVELENGTH-RESOLUTION SAR INCOHERENT Change Detection Method}

A simple processing scheme for wavelength-resolution SAR change detection based on image stack is proposed in Fig. 1. The inputs are the magnitude SAR images associated with three illuminations with no change between two of them. The

$$
\begin{gathered}
P\left(\mathbf{z} \mid H_{0}\right)=\frac{1}{2 \pi \sigma_{r} \sigma_{u} \sqrt{1-\rho^{2}}} \exp \left(-\frac{1}{2\left(1-\rho^{2}\right)}\left[\frac{\left(z_{r}-\mu_{r}\right)^{2}}{\sigma_{r}^{2}}+\frac{\left(z_{u}-\mu_{u}\right)^{2}}{\sigma_{u}^{2}}-\frac{2 \rho\left(z_{r}-\mu_{r}\right)\left(z_{u}-\mu_{u}\right)}{\sigma_{r} \sigma_{u}}\right]\right) \\
P\left(\mathbf{z} \mid H_{1}\right)=\frac{1}{2 \pi \sigma_{r} \sigma_{u} \sqrt{1-\rho^{2}}} \exp \left(-\frac{1}{2\left(1-\rho^{2}\right)}\left[\frac{\left(z_{r}-\mu_{r}\right)^{2}}{\sigma_{r}^{2}}+\frac{\left(z_{u}-\mu_{u}-s_{u}\right)^{2}}{\sigma_{u}^{2}}-\frac{2 \rho\left(z_{r}-\mu_{r}\right)\left(z_{u}-\mu_{u}-s_{u}\right)}{\sigma_{r} \sigma_{u}}\right]\right)
\end{gathered}
$$




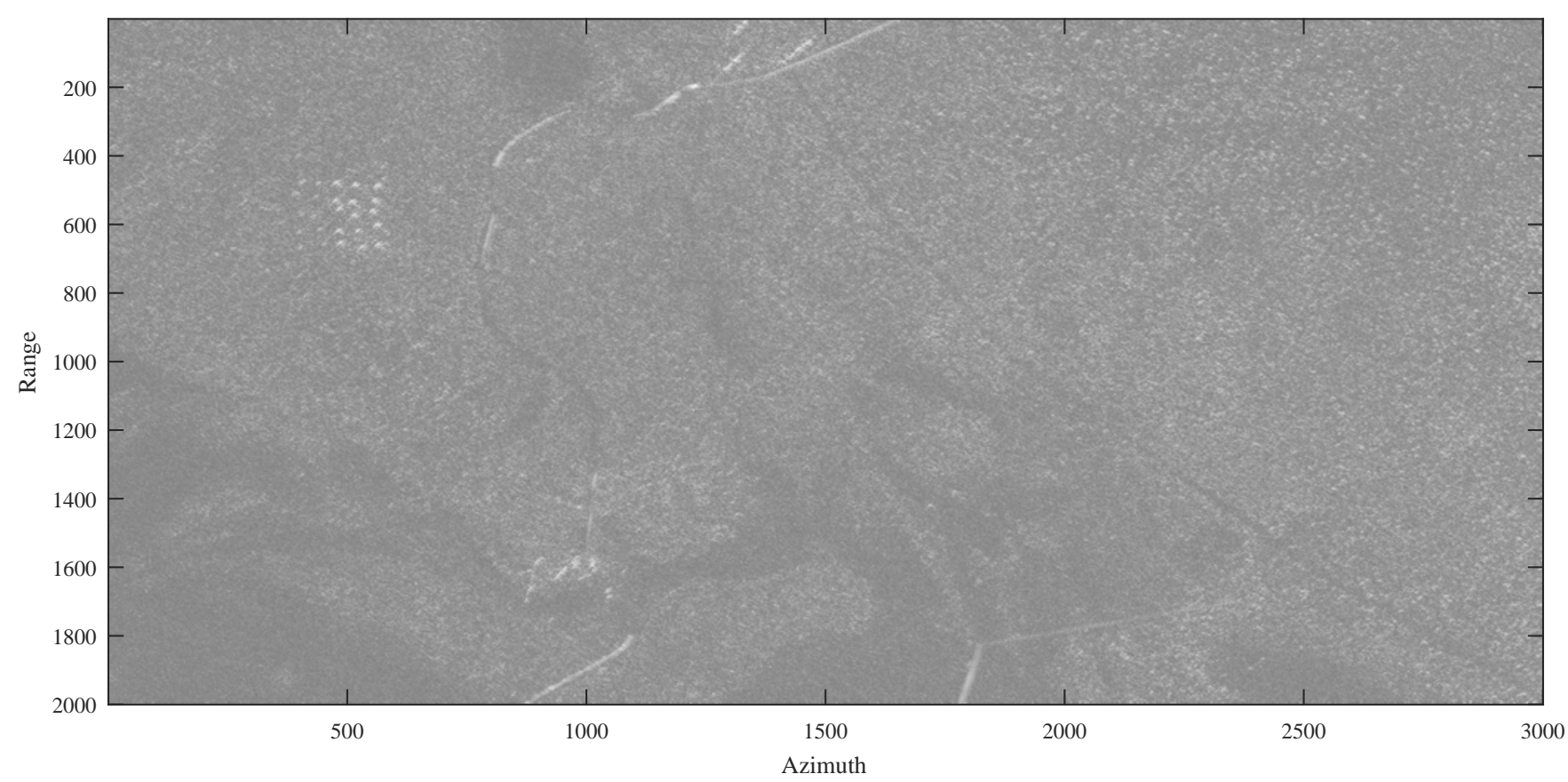

Fig. 2. Magnitude image of the ground scene with deployment of mission 3 and data was collected during pass 5.

subtraction blocks perform (1) and (2) or (3). The statistical hypothesis test is calculated using (9) or (10). By this, all images in the image stack are considered in the statistical hypothesis test. The expected values and standard deviations are calculated from whole data $\mathbf{Z}_{\mathbf{r}}$ and $\mathbf{Z}_{\mathbf{u}}$. The threshold is experimentally selected and universally applied to the statistical hypothesis test. The receiver operating characteristic curves (ROC) with thresholds are provided in the section IV-C.

Two morphological are considered in the processing scheme to minimize false alarms. The detected changes whose dimensions are below the practical spatial resolutions of SAR system are ignored. For example, the practical spatial resolutions of CARABAS in this measurement campaign are $2.5 \mathrm{~m}$ in azimuth and $2.5 \mathrm{~m}$ in range as reported in [1]. With the image sample of $1 \mathrm{~m}$ in azimuth and $1 \mathrm{~m}$ in range, the detected changes less than or equal to 3 pixels in azimuth and 3 pixels in range are removed. The detected changes separated less than the practical spatial resolutions of SAR system are connected locally to form larger objects. For CARABAS, the detected changes separated less than smaller than or equal to 2 pixels in azimuth and 2 pixels in range are connected.

\section{EXPERIMENTAL RESUlTS}

In the following experiments, we consider 24 CARABAS images retrieved in the measurement campaign in northern Sweden in 2002 [1], [2]. The goal of this measurement campaign was to provide data to evaluate the performance of change detection for targets obscured by foliage. All information concerning the measurement campaign is therefore available and can be found in [1], [2].

\section{A. Data description}

There are totally four missions and they are marked by the numbers 2, 3, 4, 5. Each mission corresponds to a deployment of 25 vehicles at certain location in the SAR scene. Each deployment is measured during six passes marked by the numbers 1, 2, 3, 4, 5, 6. Each two of six passes have identical flight heading, i.e. two with a flight heading of $225^{\circ}$, two with heading $135^{\circ}$ and two with heading $230^{\circ}$. With four missions and six passes for each mission, there are totally 24 data sets. The 24 data sets are used to form 24 SAR images. Each image has been geo-referenced to the Swedish reference system RR92 and covers a scene of size $2 \mathrm{~km} \times 3 \mathrm{~km}$. The image sample is $1 \mathrm{~m} \times 1 \mathrm{~m}$ so that each image is presented in a matrix of 3000 rows and 2000 columns.

Fig. 2 shows a magnitude image of the ground scene reconstructed from the data collected during the pass 5. The vehicles were deployed in the upper left of the scene and obscured by foliage. This deployment corresponds to the mission 3. In other missions (2, 4 and 5), these vehicles are deployed in other locations and obscured also by foliage.

\section{B. Change detection result}

The selection of images for the experiments is identical to [1], [2] so that the performance of the proposed method is easy to be evaluated and compared. The images associated with the illumination 2 are selected based on the mission, the flight heading of both the illuminations 1 and 3 as shown in Table I. An example of the output of the processing scheme is provided in Fig. 3. The white rectangle marks the location of detected changes and also the location of the deployed vehicles in the mission. The detected changes beyond the area, where the vehicles were deployed in the mission, are marked by the white circle. The changes are compared to the information of the vehicle deployment for the surveillance image. If detections that are matched to the locations of the deployed vehicles, the vehicles are declared to be found. Other detected changes even 


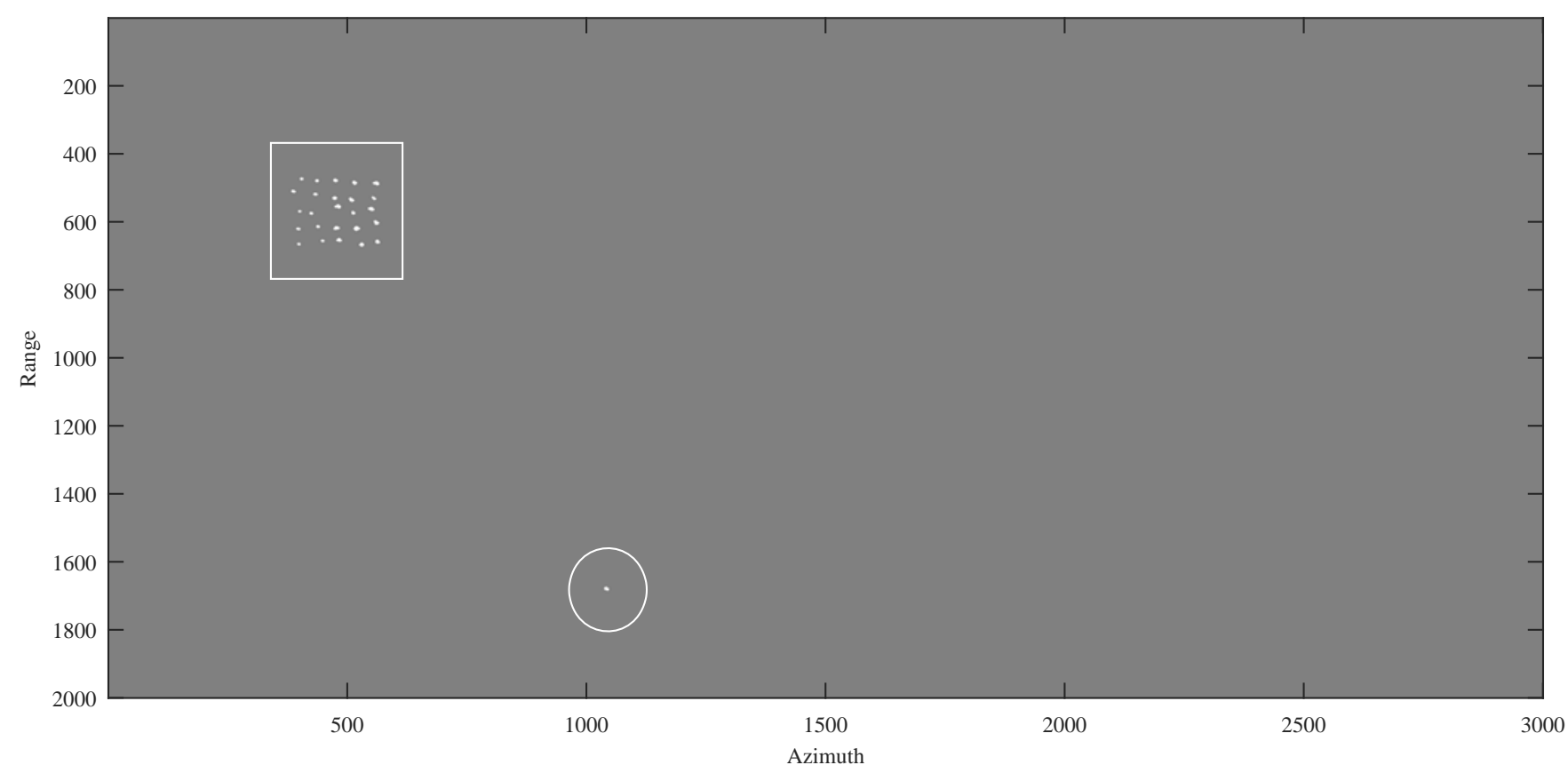

Fig. 3. An example of the output of the processing scheme where the 25 vehicles (changes) are detected (marked by white rectangle) with 1 false alarm (marked by white circle).

TABLE I

CHANGE DETECTION RESULTS OBTAINED WITH $s_{u}=0.4$ AND $\lambda=10^{4}$.

\begin{tabular}{|c|c|c|c|c|c|c|c|c|c|c|c|}
\hline \multicolumn{2}{|c|}{$\begin{array}{c}\text { Image associated } \\
\text { with illumination } 3\end{array}$} & \multicolumn{2}{|c|}{$\begin{array}{l}\text { Image associated } \\
\text { with illumination } 1\end{array}$} & \multicolumn{2}{|c|}{$\begin{array}{c}\text { Image associated } \\
\text { with illumination } 2\end{array}$} & \multirow{2}{*}{$\begin{array}{c}\text { Number of } \\
\text { known } \\
\text { targets }\end{array}$} & \multirow{2}{*}{$\begin{array}{c}\text { Number of } \\
\text { detected } \\
\text { targets }\end{array}$} & \multirow[t]{2}{*}{$P_{d}$} & \multirow{2}{*}{$\begin{array}{c}\text { Area } \\
\left(\mathrm{km}^{-2}\right)\end{array}$} & \multirow{2}{*}{$\begin{array}{c}\text { Number of } \\
\text { false } \\
\text { alarms }\end{array}$} & \multirow{2}{*}{$\begin{array}{c}\text { FAR } \\
\left(\mathrm{km}^{-2}\right)\end{array}$} \\
\hline Mission & Pass & Mission & Pass & Mission & Pass & & & & & & \\
\hline 2 & 7 & 3 & 1 & 3 & 3 & 25 & 25 & 1.00 & 6 & 0 & 0.00 \\
\hline 3 & 1 & 4 & 1 & 4 & 3 & 25 & 24 & 0.96 & 6 & 8 & 1.33 \\
\hline 4 & 1 & 5 & 1 & 5 & 3 & 25 & 25 & 1.00 & 6 & 0 & 0.00 \\
\hline 5 & 7 & 2 & 1 & 2 & 3 & 25 & 23 & 0.92 & 6 & 3 & 0.50 \\
\hline 2 & 2 & 4 & 2 & 4 & 4 & 25 & 25 & 1.00 & 6 & 0 & 0.00 \\
\hline 3 & 2 & 5 & 2 & 5 & 4 & 25 & 25 & 1.00 & 6 & 0 & 0.00 \\
\hline 4 & 2 & 2 & 2 & 2 & 4 & 25 & 25 & 1.00 & 6 & 1 & 0.17 \\
\hline 5 & 2 & 3 & 2 & 3 & 4 & 25 & 22 & 0.88 & 6 & 0 & 0.00 \\
\hline 2 & 3 & 5 & 3 & 5 & 1 & 25 & 25 & 1.00 & 6 & 3 & 0.50 \\
\hline 3 & 3 & 2 & 3 & 2 & 1 & 25 & 24 & 0.96 & 6 & 0 & 0.00 \\
\hline 4 & 3 & 3 & 3 & 3 & 1 & 25 & 25 & 1.00 & 6 & 2 & 0.33 \\
\hline 5 & 3 & 4 & 3 & 4 & 1 & 25 & 22 & 0.88 & 6 & 0 & 0.00 \\
\hline 2 & 4 & 3 & 4 & 3 & 2 & 25 & 25 & 1.00 & 6 & 0 & 0.00 \\
\hline 3 & 4 & 4 & 4 & 4 & 2 & 25 & 25 & 1.00 & 6 & 0 & 0.00 \\
\hline 4 & 4 & 5 & 4 & 5 & 2 & 25 & 25 & 1.00 & 6 & 2 & 0.33 \\
\hline 5 & 4 & 2 & 4 & 2 & 2 & 25 & 23 & 0.92 & 6 & 1 & 0.17 \\
\hline 2 & 5 & 4 & 5 & 4 & 6 & 25 & 25 & 1.00 & 6 & 0 & 0.00 \\
\hline 3 & 5 & 5 & 5 & 5 & 6 & 25 & 16 & 0.64 & 6 & 6 & 1.00 \\
\hline 4 & 5 & 2 & 5 & 2 & 6 & 25 & 25 & 1.00 & 6 & 0 & 0.00 \\
\hline 5 & 5 & 3 & 5 & 3 & 6 & 25 & 25 & 1.00 & 6 & 0 & 0.00 \\
\hline 2 & 6 & 5 & 6 & 5 & 5 & 25 & 25 & 1.00 & 6 & 0 & 0.00 \\
\hline 3 & 6 & 2 & 6 & 2 & 5 & 25 & 24 & 0.96 & 6 & 0 & 0.00 \\
\hline 4 & 6 & 3 & 6 & 3 & 5 & 25 & 25 & 1.00 & 6 & 1 & 0.17 \\
\hline 5 & 6 & 4 & 6 & 4 & 5 & 25 & 24 & 0.96 & 6 & 0 & 0.00 \\
\hline \multicolumn{6}{|c|}{ Total } & 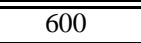 & $\bar{~} \overline{577}$ & 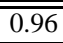 & $\bar{~} 144$ & 27 & 0.19 \\
\hline
\end{tabular}

in the deployment area that cannot be related to any vehicle are counted as false alarms. Although the deployed vehicles were controlled during the experiment there might be false alarms coming from uncontrolled or unknown changes.
Table I summarize the change detection results corresponding to a single threshold $\lambda=10^{4}$ and a single assumption of change magnitude $s_{u}=0.4$. Among 600 deployed vehicles in the mission, 577 vehicles are detected. There are totally 27 
detected objects that are either beyond the deployment areas or cannot be related to any vehicle. They are considered to be false alarms.

\section{Evaluation}

Two metrics considered for evaluating the performance of the proposed method are probability of detection $\left(P_{d}\right)$ and false alarm rate (FAR). The probability of detection is defined by the ratio of the number of detected targets to the number of known targets while the number of false alarms per square kilometer defines false alarm rate. The change detection results reported in Table I show an average probability of detection of more than $96 \%$ with less than 0.2 false alarms per square kilometer. Hence, the performance of the proposal is better than the change detection results presented in [2]. With a similar average probability of detection, the false alarm rate has been reduced with a factor of about 4 . Please note that the proposed processing scheme does not include averaging data by a low pass filter that can affect significant the change detection results.

For a better evaluation of the method and a better comparison between the proposed method and others methods that have been published, we combine the resulting average probability of detection and false alarm rate for different values of assumed magnitude $s_{u}$ and threshold $\lambda$ in ROC. ROC for the best performance obtained with both coherent and incoherent change detection methods is extracted from [1] and plotted in the same Fig. 4 as a reference. For comparison purpose, the horizontal axis is $10 \log _{10}$ (FAR) instead of FAR.

We consider here the thresholds in the range $\lambda \in$ $\left[10^{-8}, 10^{8}\right]$. For assumptions for the magnitude, we can set $P\left(z_{u}>s_{u}\right)$ as desired and calculate $s_{u}$ based on (12). In the case, the assumptions for the magnitude will change with respect to the data. For evaluation purpose, we can simply set $s_{u}=0.1,0.4$ and 0.8 .

As observed in Fig. 4, the extracted ROC from [1] lies below the others given by the proposed method. For example, for $10 \log _{10}(\mathrm{FAR})=0$, the best detection probability given by [1] is about only $90 \%$ while the proposed method provides the detection probability of about $98 \%$. For very low false alarm rate, e.g. $10 \log _{10}(\mathrm{FAR})=-1$, the detection probability given by [1] drops below $60 \%$ while the proposal still maintains the detection probability more than $90 \%$. Such an improvement in detection probability is significant.

ROCs plotted in Fig. 4 also shows how the assumptions on magnitude affect the performance of the proposal. The difference in detection probability is only about $1 \%$. Hence, assumptions for the magnitude $s_{u}$ are not very sensitive to the performance of the proposal. As observed, the best performance is associated with $s_{u}=0.4$ where the assumption on magnitude is close to the true average value $(\approx 0.5)$. When $s_{u}=0.8$, several vehicles with low radar cross-section are undetected. The detection probability is always below $100 \%$ inspire of very high false alarm rate, e.g. $10 \log _{10}(\mathrm{FAR})=2$. This corresponds to the case that the assumption on magnitude is higher than the magnitudes of certain vehicles. The samples associated with those vehicles will not be considered in the

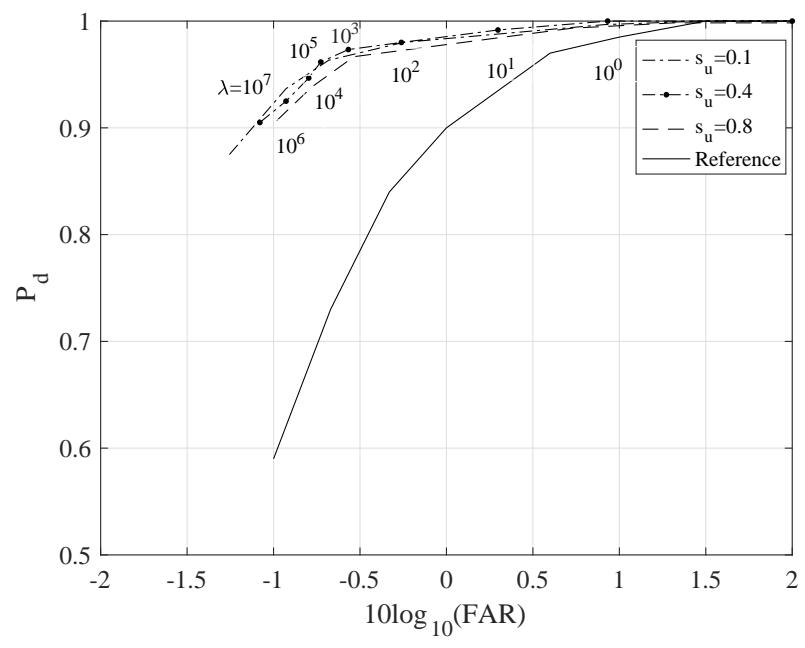

Fig. 4. ROCs obtained with the proposal compared with best ROC extracted from [1]. The selected thresholds $\left[10^{0}, 10^{7}\right]$ for the case $s_{u}=0.4$ are provided.

statistical hypothesis test and the vehicles cannot be detected. The performance for the case of $s_{u}=0.1$ is not very different from others. However, the detection probability reaches $100 \%$ with very high false alarm rate $10 \log _{10}(\mathrm{FAR}) \approx 1.5$.

\section{CONCLUSION}

A wavelength-resolution SAR incoherent change detection method based on image stack is introduced in this paper. Considering image stack in statistical hypothesis test for change detection results into a simple mathematical expression and provide very good change detection results. Hence, statistical hypothesis test derived in this paper is presented in a simple form and easy to implement. An average detection probability of $96 \%$ with a false alarm rate of only 0.19 per square kilometer is an example of the performance of the proposed method. The improvement in average detection probability as well as the reduction in false alarm rate are significant in comparison to the published methods.

\section{REFERENCES}

[1] L.M.H. Ulander, M. Lundberg, W. Pierson, and A. Gustavsson, "Change detection for low-frequency SAR ground surveillance", IEE Proc.-Radar Sonar Navig., vol. 152, no. 6, pp. 413-420, 2005.

[2] M. Lundberg, L.M.H. Ulander, W.E. Pierson and A. Gustavsson, "A challenge problem for detection of targets in foliage", in Proc. SPIE Algorithms for Synthetic Aperture Radar Imagery XIII, vol. 6327, Orlando, FL, April 2006, pp. (13p).

[3] V.T. Vu, R. Machado, M.I. Pettersson, P. Dammert and H. Hellsten, "False alarm reduction in wavelength-resolution SAR change detection using adaptive noise canceler", IEEE Trans. Geosci. Remote Sensing, vol. 55, no. 1, pp. 591-599, 2017.

[4] A. Anees and J. Aryal, "A statistical framework for near-real time detection of beetle infestation in pine forests using MODIS data", IEEE Geosci. Remote Sensing Lett., vol. 11, no. 10, pp. 1717-1721, 2014.

[5] A. Anees and J. Aryal, "Near-real time detection of beetle infestation in pine forests using MODIS data", IEEE Journal of Selected Topics in Applied Earth Observations and Remote Sensing, vol. 7, no. 9, pp. 3713-3723, 2014.

[6] R. Machado, M.I. Pettersson, V.T. Vu, P. Dammert and H. Hellsten, "The stability of UWB low-frequency SAR images", IEEE Geosci. Remote Sensing Lett., vol. 13, no. 8, pp. 1114-1118, 2016. 